\title{
LA PROTECTION DES CONDUITES FORCÉES
}

\author{
M. Bernard DAUTRUY \\ Directeur de E.M.I.
}

La profection des conduites forces neuves ainsi que l'entretien des conduites déjà en service pose de nombreux problemes sur lesquels se penchent les techniciens. Ces problemes sont les mèmes d'ailleurs que l'on retrouve pour les vannes, les batardeaux, les grilles de prise d'eau, etc.

Le problème capital est l'application, à l'intérieur ou a l'extéricur de la conduite, d'une couche protectrice durable, soit de peinture, soit de revètement similaire. En effei, non seulement les travaux concernant la protection sont d'un prix élevé, mais l'arrêt des lurbines pour l'entretien de la conduice est préjudiciable au rendement du réseau. Aussi, les techniciens demandent-ils aux fabricants de peintures ou aux applicateurs de lear foumir une couche protectrice capable de préserver les conduites pendant de nombreuses années.

Les premieres protections consistaient en peintures anti-rouille à base d huile, mais potir les partics d'une construction plongées toujours ou partiellement dans l'eau, une peinture à base d'huile se gonfle, n'adhère plus exacıement à la paroi et, dans ces conditions n'empèche plus l'attaque de la rouille.

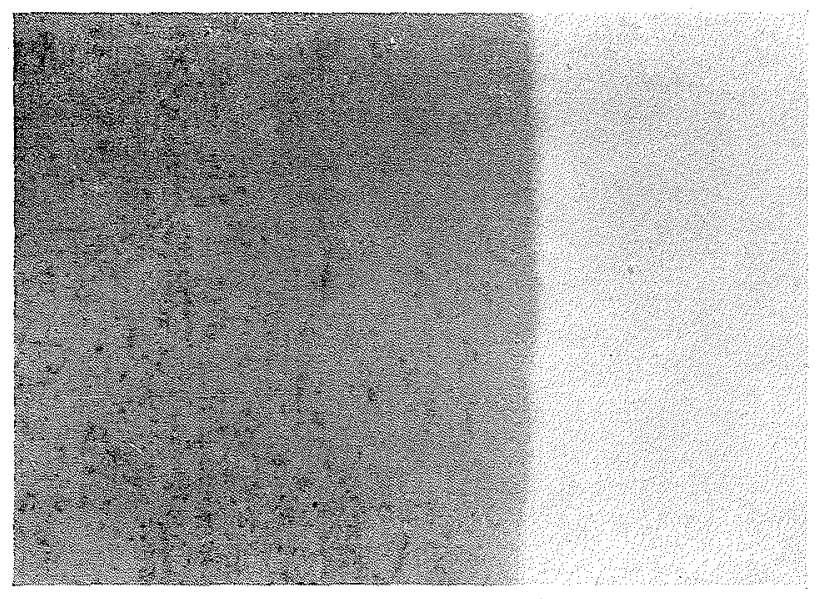

Fig. 1
Une technique plus récente fait usage de peinture à base de bitume et depuis quelques années, certaines maisons françaises et étrangères onl mis au point des peintures bitumineuses s'appliquant à froid ou à chaud et domant toutes, dans la plupart des cas, de très bons résultais; ceuxci, cependant, ne sont pas encore suffisants par rapport au prix élevé de la peinture et de son application, ainsi que de la perte due à l'arrêt occasionné par les travaux.

Il y a donc fallu chercher à améliorer la durée de protection par une meilleure application, ou plutot par un meilleur «accrochage » de cetle couche de protection, car l'usage a montré, comme nous larons vu dans le cas des peintures it base d'huile, que c'est ladhérence totate de ia peinture qui est le pont crucial de la protection.

Jusqu'ici, lorsquil sagissait de repeindre l'incérteur ou lextérieur a une conduite forcée nenve, les applicateurs dans la plupart des cas, se contentarent de brosser les parois, le metal etant « propre " par dérinition.

Lorsqu il sagissat de conciuites déjà anciennes ou la rouille torme épaissear, la pus grosse partie de celle-ci était enlevée par piquetage à mains, ou pneumatique, puis la conduite était

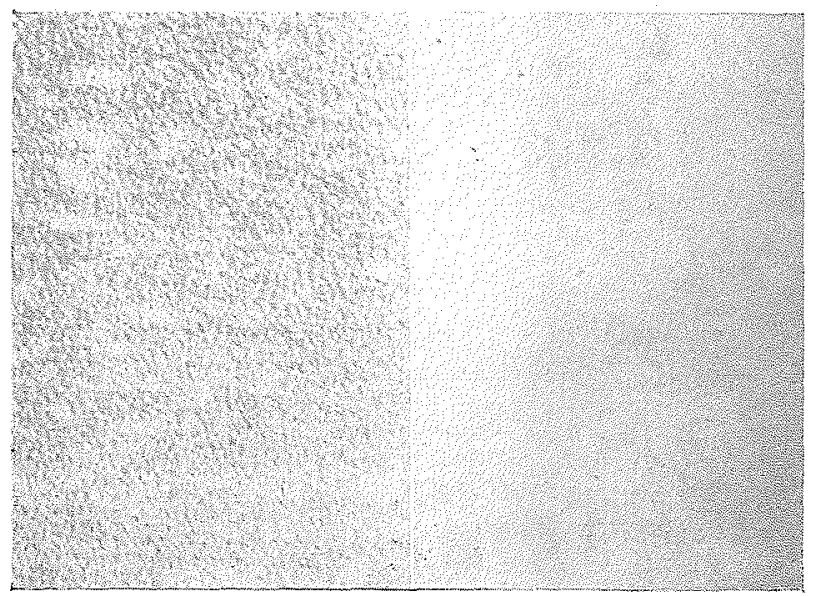

Fig. 1 bis

CONDUTTE ROUHLÉ AYANT ET APRES SABLAGE 


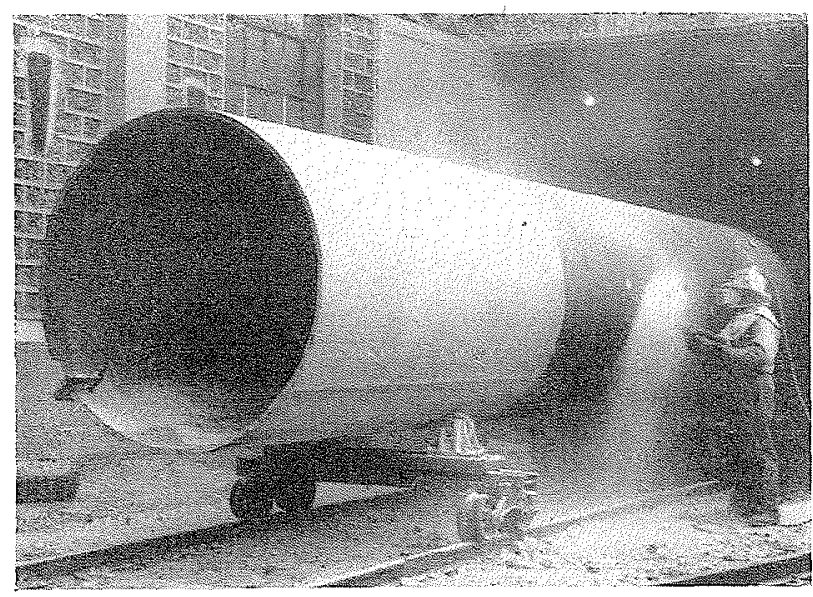

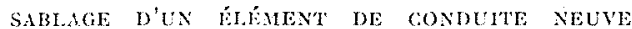

TRAVAL EN USINE

brossé ; mais ces procédés n’arrachaient nullement la rotille ancrée dans le métal.

Le piquetage enlève la surépaisseur de rouille ou de tartre se trouvani sur les parois, le brossage enleve lautre partie de cette oxydation, mais le mécial qui se ronge à sa surface se troave poli par le brossage. Le travail de décapage est done à moitié réalisé et même si le passage de la brosse est fait avec soin, la couche de rouille est polie et pénètre dans le métal.

Ia couche de peinture est donc passée sur une sturace mal preparée ei quelle que soit la qualits l'e cette peinture, au bout d'un certain temps, l'oxydation restante reprend, sous l'action de l'humidié, son travail sournois, et l'on s'apesçoit que la couche protectrice se décolle $\mathrm{par}$ plaques et que mème à certains endroits, la rouille ronge le métal sous la peinture et afraiblit la paroi au poini qu'il a fallu parfois envisager le remplacement de certains élóments de conduites.

Lorsqu'il s'agit de conduites neuves, la rouille n'existe pratiquement pas; mais il reste par endrois la pellicule de laminage qu'il n'est pas possible d'enlever soit à la brosse à main, soit à la brosse pneumatique. Comme dans le cas précedent, la brosse aura une action de polissage et c'est sur ce poli que viendra s'appliquer la peiniture qui n'aura aucun accrochage; sous laction de l'humidite, la pellicule de laminage se dćcollera, enirainant avec elle la peinture, quelie que soit la qualité de celie-ci.

Afin d'obienir de bons résultats, il faut que ia couche de protection augmente sa durée par son accrochage à la paroi et seule une bonne préparaion de décapage peut amener ce résultat. Il faut donc que les parois de la conduite soient aussi nettes que possible, ne présentant aucune trace d'oxydation, et mìme lorsque cela est possible, aucune trace de poussiere. Celle-ci, en effet, aspire l'humidite de l'air et une peinture passée sur celte poussiere humide ne tardera pas à subir les facheuses conséquences font nous avons parlé plus haut.

Comme une peinture ne se fixe que difficilement sur une surface libre, il faut que le métal sur lequel on l'applique soit rugueux afin qu'elle s'y accroche et pénètre la surface des parois à enduire; ainsi done la rouille ne pourra s'interposer entre le métal et la peinlure.

Il est indiscutable qu"en opérant ainsi et en utilisant une bonne couche de protection, la conduite sera préservée pour une durée beaucour) plus grande. La pratique a montré que le sablage de la conduite avant lapplicaion de la couche de protection permet dobtenir un résultal excellent; ce sablage peut cire réalise avec plus ou moins de difficultés dans tous les cas, malgré le diametre et l'inclinaison de la conduite, à condition d'adapter un matériel puissani et de le rendre apte à servir aux travaux intérieurs des conduites forcées, surtout si les trous d'hommes manquent on s'ils sont tres éloignés. Il y a lien, également, d'utiliser toujours un mème personnel, rompu à ce genre de travaux; certains spécialistes de ce travan, en conduites, arrivent a sabler une surface aussi grande qu'ils pourraient le faire en acelier.

Dans certains cas, sur des conduites très longues, le sabletar est en relation téléphonique au fur et à mesure de son avancement avec la personne chargée de faire mancuver la sableuse. Le travail peut done se fare d'une maniere ininterrompue, le sableur recevant des ordres au moyen découteurs places sous son casque, le fil du téléphone étant protégé sinon invisible, à laute d'ètre décortiqué par le jet de sable ou les ricochets.

Un point important est l'étude de la qualité du sable utilisé pour le travail; ce sable doit cure abrasif, afin d'obtenir un bon résultat; if doit ètre également lavé, séché, dépoussiéré ec calibré. Le séchage doit s'en effectuer aux courants d'air chaud et non sur plaques, ce dernier procédé brùlant le sable qui s'ecrase ensuite très facilement a la projection, ne cree pas dasperités et se réduit en poussière.

Le traitement du sable qui doit servir à ce genre de traval demande beaucoup de soins, puisque d'un sable de grosseur moyenne indépen. damment de la poussière que l'on peut en rebirer, on extrait normalement $200 \mathrm{kgs}$ d'eau pour $1500 \mathrm{kgs}$ de sable de grosseur moyenne (16-14). Le mélange de sable servant à décaper, se fai suivant le degré de profondeur de la couche 
d'oxydation. Le sable de rivière ou de mer est à déconseiller, car n'étant pas de pure silice et conienant trop de poussières, il n'est pas abrasif.

Le sablage, indépendamment de la propriété qu’il possède de débarrasser le métal de la rouille, a l'arantage de décaper partout ou l'outil grattoir, boucharde ou brosse) ne peut passer.

Il est tres rare, surtout lorsqu'on sable und vanne, de ne pouroir eflectuer le travail enliorement, étant donné que dans certains cas très difficiles, le sablage peut se faire par ricochets.

Un autre arantage du sablage est de déceler les parties de la conduite rendues faibles par l'oxydation ; il est arrive mème que le jet passe a travers le métal quand celui-ci est en mauvais état, mais mème lorsque le métal est afraibii, on peut toujours le recharger après le travail du sableur. Une autre propriéié du sablage est de permettre la vérification des soudures beaucoup plus nettement après le passage du jet.

Dans certains cas, l'usage a montré que lorsqu'une peinture est appliquće après sablage, sal aurée est double; ce mode de décapage tend a se généraliser de plus en plus el dans certains Pays, notammene la Suisse, beaucoup de conduites forcées' ont subi ces traitements avant l'application de la peinture de protection ; les condutes de Vernayaz, d'Aproz, de Dixence, de Ganierbach en sont des exemples très connus et dont les résultats sont excellents.

Cette protection des conduites par l'emploi de la peinture dont nous venons de parler, n'est pas le seul genre de protecion spécialisce et il existe actuellement un autre genre de protection très efficace, dont la durée est de beaucoup supérieure a la peinture: ce procédé que l'on applique

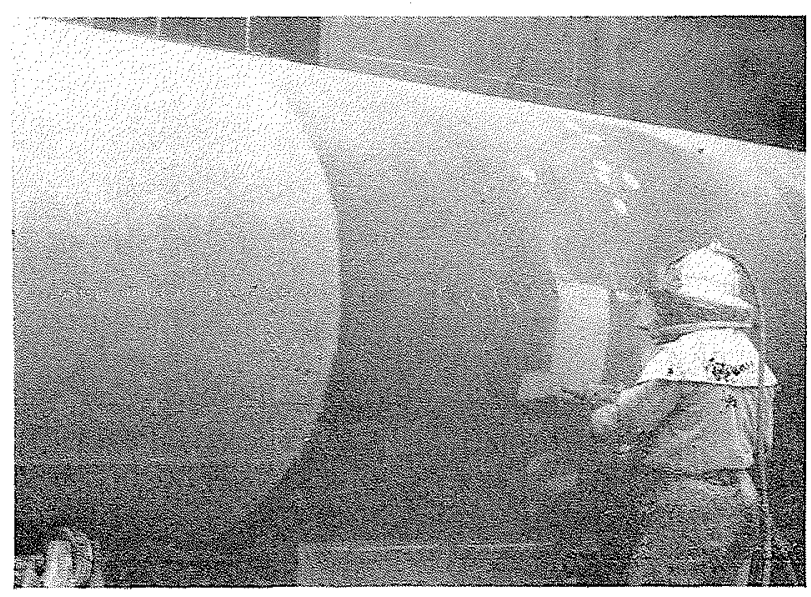

SABLAGE D'UNE CONDUTE

(SLRFACE DU MÉTAL COMPLETEMENT DÉBARRASSÉE DE LA ROUILLE)

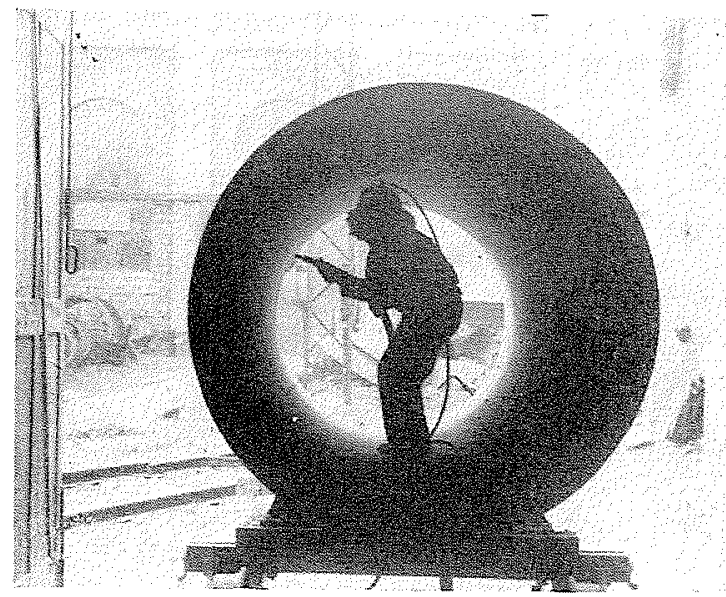

TRAVAIL A L'IN'TERIEUR D'UNE CONDUITE

surtoul aux conduites forcées neuves est la Métallisation, c'est-à-dire la protection du métal par un autre métal ou deux métaux. Le berceau de ce procédé, qui rend des services aussi bien comme protection que comme rechargement, est la Suistse, où l'inventeur Schoop l'a mis en application en 1910 ; la métallisation est actuellement utilisée à peu près partout et son emploi est très répandu.

l'ar ce procédé, les conduites forcées sont en quelque sorte doublées d'une armature de protection en un métal ne rouilant pas au contact de l'eau, le zinc étant le plus employé.

Cette armature fait corps avec la conduite et clle est comme la peinture employée précédemmeni, ancrée dans lacier de la conduite. Le Schoopage ou métallisation d'une conduite se fait au moment de sa construction chez le fabricant, ou sur le chantier avant la pose de chaque élément.

Avani de commencer le Schoopage, il y a lieu de connaître la composition de l'eau devant passer par la conduite. A cet elfet, deux analyses doivent être faites, l'une au plus bas débit des eaux, l'autre au plus haul, car suivant les couches géologiques traversées par l'eau et le débil de celle-ci, sa composition varie et le résultat final de ces analyses pourra seul déterminer l'épaisseur de la couche de proteciion à appliquer. Son épaisseur peut varier de $12 / 100$ à 7 ou $8 / 10$.

Avant la métallisation, les éléments de la conduite seront sablés blanc argent au sable calibré siliceux dont te grain devra correspondre proportionnellement au grain du métal ulilisé.

La projection du sable crée des alvéoles assez profonds dans le métal, alvéoles dans lesquels la partie sous-jacente du métal de protection projeté en fusion sous 4 ou $5 \mathrm{kgs}$ de pression 
viendra se loger, donnant une adhérence parfaite à la couche par incrustation dans le métal support.

Pour faciliter le montage des éléments de conduite, chaque élément esí métallisé sur toute sa surface, sauf une largeur denviron $20 \mathrm{~cm}$. du bord, pour permettre la soudure d'assemblage ; après soudure, il ne restera que la métallisation des raccords, métallisation qui sera rapidement effectuée.

La protection de l'intérieur des conduites peut ètre augmentée par l'application sur la première couche de zinc de base de deux couches d'aluminium qui seront polies afin de n'ofirrir à l'eau aucune résistance; pour l'extérieur de la conduite, 2 ou 3 couches de zinc recouvertes d'une couche de peinlure suffiront à sa protection pour de très longues années.

Toujours suivant les endroits, cette durée de protection peut être deux fois celle de la peinture appliquée normalement.

Etant donné la rapidité des travaux d'application, la conduite peut être livrée métallisée et polie, sauf les raccords qui seront effectués sur le chantier. D'autre part, le Schoopage offre un gain de temps appréciable puisque immédiatement après la métallisation, l'eau peut circuler dans la conduite sans dommage.

Enfin, le prix de revient de cetle protection est sensiblement plus économique que celui d'une application de peinture, 5 à $10 \%$ meilleur marché pour les conduites neuves.

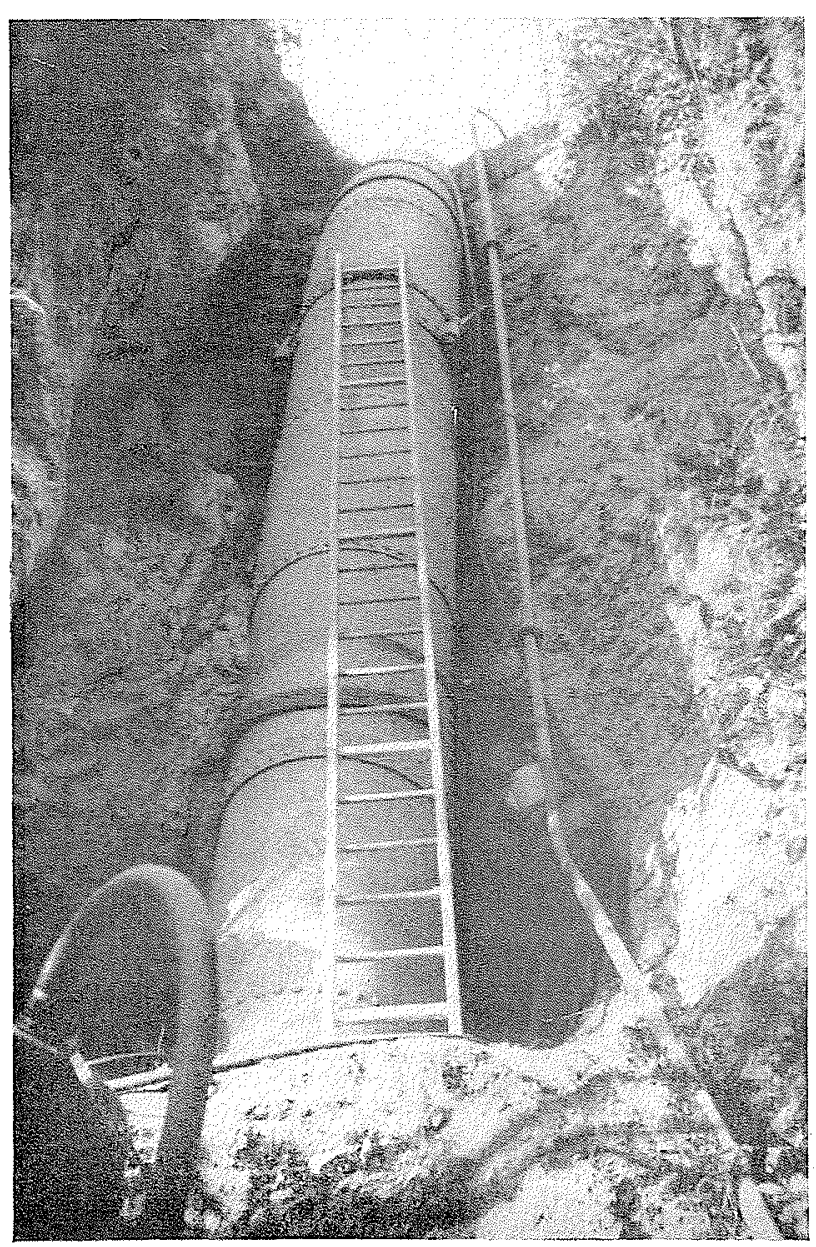

TRAVAL SUR CHANTLR (PONT-DU-LOUP) 